\title{
Characterization of Serratia isolates from soil, ecological implications and transfer of Serratia proteamaculans subsp. quinovora Grimont et al. 1983 to Serratia quinivorans corrig., sp. nov.
}

${ }^{1}$ Cardiff School of Biosciences, Cardiff University, PO Box 915, Cardiff CF10 3TL, UK

2 Centre for Ecology and Hydrology - Oxford, Mansfield Road, Oxford OX1 3SR, UK

\author{
Kevin E. Ashelford, ${ }^{1}$ John C. Fry, ${ }^{1}$ Mark J. Bailey ${ }^{2}$ and Martin J. Day ${ }^{1}$ \\ Author for correspondence: John C. Fry. Tel: +44292087 4190. Fax: +442920874305. \\ e-mail: fry@cardiff.ac.uk
}

\begin{abstract}
Eleven strains of Serratia were isolated from different soils and the guts of invertebrates and characterized by their sensitivity to eight indigenous bacteriophages. They were also classified according to bacteriocin production and sensitivity, BiOLOG plate and API 20E strip profiles and 16S rRNA sequence information. One strain was thus identified as Serratia plymuthica, another as Serratia fonticola. The remaining strains were shown to be closely related to Serratia proteamaculans subsp. quinovora Grimont et al. 1983 after DNA-DNA cross-hybridization demonstrated relatedness greater than $70 \%$ with the type strain of this subspecies. From an ecological perspective, our results illustrated the wide variation in sensitivity that closely related Serratia strains have towards various indigenous soil phages and that these phages have broad host ranges within the genus. Furthermore, the phage and bacteriocin interactions within the Serratia strains examined were intricate and did not reflect phylogenetic relationships. These results together imply that complex interactions will occur in soil within the natural community of Serratia strains and their bacteriophages. DNA-DNA cross-hybridization and phenotypic characterization showed that $S$. proteamaculans subsp. quinovora strains formed a cohesive group at the species level. It is therefore concluded that these strains should be designated as Serratia quinivorans corrig., sp. nov.
\end{abstract}

Keywords: Serratia, DNA-DNA hybridization, 16S rRNA gene, bacteriophages, bacteriocins

\section{INTRODUCTION}

Serratia appears to be a ubiquitous genus in nature, and ten species are currently recognized (Grimont \& Grimont, 1992). Serratia species have been isolated from water, soil, animals (including man) and from the surfaces of plants (Grimont \& Grimont, 1992). Typical phytosphere isolates belong to the 'Serratia liquefaciens-proteamaculans-grimesii' complex (Grimont et al., 1981), also called S. liquefaciens-like

Full tables of results for bacteriocin production and DNA-DNA crossreaction for the novel isolates are available as supplementary material in IJSEM Online (http://ijs.sgmjournals.org).

Abbreviations: FAME, fatty acid methyl ester; SSM, Serratia selective medium; TSBA, tryptone soy broth agar.

The DDBJ/EMBL/GenBank accession numbers for the $16 \mathrm{~S}$ rRNA sequences analysed in this paper are AJ279044-AJ279055. species (Grimont et al., 1982b). Strains of these species produce plant-growth-promoting chemicals, have anti-fungal properties, encourage the establishment of nitrogen-fixing symbionts and act as insect pathogens (Grimont \& Grimont, 1992; Kalbe et al., 1996; Zhang et al., 1996, 1997). A greater understanding of the ecology of these bacteria is required if the impact of Serratia species on agriculture is to be adequately understood and, if necessary, manipulated. A potentially important controlling factor for bacterial populations is predation by bacteriophages (phages).

In previous studies, we characterized a population of phages that predated on indigenous Serratia strains in the rhizosphere of sugar beet (Ashelford et al., 1999a, b, 2000). This phage population was complex, both in terms of its temporal population dynamics and its diversity. The host species studied was a rhizosphere-derived strain named CP6 and originally 
identified by fatty acid methyl ester (FAME) analysis as $S$. liquefaciens. In the current study, we aim to describe the isolation, enumeration, characterization and biodiversity of further examples of this bacterium from the same environment and from two other sites. Furthermore, we also investigate the identification and taxonomy of these isolates within the current understanding of the genus Serratia as a whole.

\section{METHODS}

Bacteria and bacteriophages. Bacteria used in this study (Table 1) were stored in $50 \%$ glycerol at $-80{ }^{\circ} \mathrm{C}$ and maintained on nutrient agar (Oxoid, CM3). The phages used were ФСР6-1 to ФСР6-6 (Ashelford et al., 1999b) and two further phages, ФСР6-8 and ФСР6-9, isolated during this study. Fresh phage lysates were prepared by co-inoculating nutrient broth with phage and host bacterium and incubating overnight with agitation at $20^{\circ} \mathrm{C}$. Cultures were centrifuged for $10 \mathrm{~min}$ at 4000 r.p.m. and their supernatants were filtered $(0.22 \mu \mathrm{m}$ pore size $)$ to produce final lysates, which were stored at $4{ }^{\circ} \mathrm{C}$ or under $50 \%$ glycerol at $-80{ }^{\circ} \mathrm{C}$. CP6 (Ashelford et al., 1999a) was used as the host bacterium for all phages except for ФСР6-8 and ФCP6-9, where strains CP6b and CP6d, respectively, were used. Phage lysates were titrated with the overlay-agar method of Adams (1959) using $1.3 \%(\mathrm{w} / \mathrm{v})$ nutrient broth (Oxoid, CM1) solidified with $0.65 \%(\mathrm{w} / \mathrm{v})$ agar (Oxoid, L11) as overlay on a nutrient agar base.

Isolating CP6-like bacteria from the environment. A crop of sugar beet (Beta vulgaris var. Amethyst) was grown at Wytham Farm, Oxford, from seeds (EB3 pellets; Germains UK, Kings Lynn, UK) sown on 16 May 1997. The resulting plants were sampled and homogenized as described previously (Ashelford et al., 1999a). Fresh and freezer-stored homogenates were plated onto Serratia selective medium (SSM; Ashelford et al., 2000) and incubated for $48 \mathrm{~h}$ at $30{ }^{\circ} \mathrm{C}$ to select for bacteria that resembled CP6. Estimates of total viable heterotrophic bacteria were obtained by plating serial dilutions of the homogenates onto tryptone soya broth agar (TSBA) incubated for 7 days at $15^{\circ} \mathrm{C}$. Three separate leaf-litter samples were collected from Oxleas wood, a deciduous woodland in Kent, UK, and homogenates were plated onto SSM. Invertebrates in the samples, and the gut contents of earthworms from scrubland in Cornwall, were also homogenized and plated onto SSM.

Testing CP6-like colonies for phage sensitivity. Colonies on SSM plates with the typical CP6 morphology (1 mm orange colonies with yellow borders) were purified on TSBA and screened for phage sensitivity using overlay-agar plates. Phage lysates ( $5 \mu 1$; ФСР6-1 to -9) were dropped onto each plate and examined for zones of lysis after overnight incubation at $15^{\circ} \mathrm{C}$. CP6-like isolates were grouped by sensitivity to the phage lysates and representatives of these 'sensitivity-groups' were selected for analysis. In addition, two representative isolates that appeared CP6-like on SSM but not on TSBA were also studied further.

Phage sensitivity of CP6 isolates and various Serratia species representatives (Table 1) was quantified using fresh overlayagar plates. Lysates were serially diluted and drop-plated (10 $\mu 1)$ in triplicate onto these plates and incubated overnight at $15^{\circ} \mathrm{C}$ and plaques were counted. All titrations were done in duplicate.

Assessing the host range of bacteriocins produced by the strains. All bacteria were checked for bacteriocin production and lysogeny. Nutrient-broth cultures were grown overnight under constant agitation at $20^{\circ} \mathrm{C}$ and $100 \mu$ was used to inoculate overlay-agar plates. The remainder was centrifuged at 4000 r.p.m. for $10 \mathrm{~min}$ and the supernatant passed through a $0.22 \mu \mathrm{m}$-pore filter. Each filtered supernatant was then drop-plated $(5 \mu \mathrm{l})$ onto the overlay plates. Plates were scored after overnight incubation at $15^{\circ} \mathrm{C}$ for the presence of zones of lysis. To confirm that lysis was not due to phage infection, the supernatants were serially diluted and plated to confirm that the zones would not resolve into individual plaques.

Probing bacterial DNA with DIG-labelled phage probes. DNA was extracted from phages ФCP6-1 to -6 by a proteinase-K method (Day \& Marchesi, 1996) and resuspended in TE buffer (Sambrook et al., 1989). DNA was digested with EcoRI according to the manufacturer's instructions (Promega), purified with buffered phenol/chloroform/ isoamyl alcohol (25:24:1; Sambrook et al., 1989) and randomly labelled with DIG using DIG-High Prime

Table 1. Bacterial strains used in this study

Culture collections are abbreviated as: ATCC, American Type Culture Collection; BCCM, Belgian Co-ordinated Collections of Microorganisms; CIP, Collection of bacterial strains of Institut Pasteur; NCIB, National Collection of Industrial Bacteria; NCTC, National Collection of Type Cultures.

\begin{tabular}{|c|c|c|}
\hline Strain(s) & Comments & Source \\
\hline CP6 (= NCTC 13188) & Sugar-beet isolate from Wytham Farm, Oxford & Ashelford et al. (1999a) \\
\hline CP6a, CP6b, CP6c, CP6d, CP6e ( = NCTC 13190), CP6f, CP6g & Sugar-beet isolates from Wytham Farm, Oxford & This study \\
\hline $\mathrm{PC} 1(=$ NCTC 13191$)$ & Earthworm-gut isolate from scrubland, Cornwall & This study \\
\hline RK (= NCTC 13192) & Sugar-beet isolate from Wytham Farm, Oxford & This study \\
\hline Ox1 & Leaf-litter isolate from Oxleas wood, Kent & This study \\
\hline Ox2 (= NCTC 13194) & Invertebrate-gut isolate from Oxleas wood, Kent & This study \\
\hline Serratia marcescens NCIB 1377 & & NCIB \\
\hline Serratia ficaria $\mathrm{NCTC} 12148^{\mathrm{T}}$ & Type strain & NCTC \\
\hline Serratia liquefaciens CIP $103238^{\mathrm{T}}$ & Type strain & NCTC \\
\hline Serratia proteamaculans subsp. proteamaculans CIP $103236^{\mathrm{T}}$ & Type strain & NCTC \\
\hline Serratia proteamaculans subsp. quinovora $\mathrm{LMG} 7887^{\mathrm{T}}$ & Type strain & $\mathrm{BCCM}$ \\
\hline Serratia grimesii CIP $103361^{\mathrm{T}}$ & Type strain & $\mathrm{BCCM}$ \\
\hline Serratia plymuthica CIP $103239^{\mathrm{T}}$ & Type strain & CIP \\
\hline Serratia fonticola IB4r ( = NCTC 13193) & Isolated from pilot-scale sewage bed & Ashelford et al. (2001) \\
\hline Serratia fonticola $\mathrm{LMG} 7882^{\mathrm{T}}$ & Type strain & $\mathrm{BCCM}$ \\
\hline Hafnia alvei $\mathrm{NCTC} 8105^{\mathrm{T}}$ & Type strain & NCTC \\
\hline
\end{tabular}


(Roche). Labelled DNA was then mixed together, in equal proportions, to produce a final DIG-labelled phage-DNA cocktail containing all six CP6 phages.

DNA was extracted from all bacteria using the cetyltrimethylammonium bromide (CTAB) method of Powell et al. (1993). DNA from four CP6 lysogens (CP6-ly- Ф1, CP6ly-Ф2, CP6-ly-Ф3, CP6-ly-Ф5; Ashelford et al., 1999b) was extracted as positive controls. DNA extracts were transferred onto positively charged nylon membrane (Roche) using a slot-blotter and the membrane was probed with the DIG-labelled phage DNA at $68^{\circ} \mathrm{C}$ using standard hybridization buffer $[5 \times \mathrm{SSC} ; 1 \%(\mathrm{w} / \mathrm{v})$ blocking reagent; $0 \cdot 1 \%(\mathrm{w} / \mathrm{v}) N$-lauroylsarcosine, sodium salt; $0.02 \%$ SDS] following the manufacturer's methodology.

Phenotype-based identification of representative isolates. All strains (Table 1) were characterized phenotypically using the API 20E (bioMérieux) and BiOLOG GN microplate (Biolog) identification systems. The sugar-beet isolates CP6 and $\mathrm{CP} 6 \mathrm{a}-\mathrm{CP} 6 \mathrm{~g}$ were also characterized in triplicate by FAME and cluster analysis. Isolates CP6 and CP6e were additionally characterized using the Biotype 100 system (bioMérieux). This was undertaken for the authors by the BCCM/LMG (Belgian Co-ordinated Collections of Microorganisms; Laboratorium voor Microbiologie, Universiteit Gent, Belgium) in conjunction with Professor P. A. D. Grimont. Biotype growth profiles were compared to the 'Enterobacteriaceae '94' database and strain identities with Recognizer (Institut Pasteur Taxolab). CP6-like isolates and some type strains of Serratia species were also tested for growth on M70 minimal agar with $0 \cdot 1 \%$ (w/v) quinic acid (Sigma) at $30{ }^{\circ} \mathrm{C}$ for 14 days (Grimont \& Grimont, 1984).

16S rRNA amplification and sequencing. Genomic DNA was extracted using the CTAB method and amplified by PCR with primers 63f (5'-CAGGCCTAACACATGCAAGTC$\left.3^{\prime}\right)$ and 1387r (5'-GGGCGGWGTGTACAAGGC-3') (Marchesi et al., 1998). The PCR products (approx. $1300 \mathrm{bp}$ ) were cloned using the pGEM-T Easy vector system (Promega) and sequenced from each end with M13 primers on a LI-COR DNA4000L sequencer (MWG-Biotech), using fluorescent-primer labelling with near-infrared IRD800, according to the manufacturer's instructions.

Phylogenetic analysis of $16 \mathrm{~S}$ rRNA sequences. Sequences were aligned with those of Serratia and other members of the Enterobacteriaceae from the DDBJ/EMBL/GenBank databases with CLUSTAL W (Thompson et al., 1994). Only sequences $>1300 \mathrm{bp}$ were used and we ensured that a representative of each available enterobacterial genus was included in our analysis. After editing the aligned dataset to remove gaps, unknown bases and obvious mismatches, evolutionary distances were calculated with the Jukes and Cantor algorithm. Phylogenetic trees were determined by the neighbour-joining method using TREECON for Windows (Van de Peer \& De Wachter, 1994). For comparison, additional phylogenetic trees were constructed using other tree-estimation methods. The Fitch-Margoliash method was applied to Jukes and Cantor-derived distance matrices using the programs FITCH and KITSCH of the PHYLIP computer package (Felsenstein, 1993). From the same package, the programs DNAML and DNAMLK were also used to calculate tree topology by maximum-likelihood, whilst DNAPARS was used for parsimony analysis.

DNA-DNA cross-hybridization. This was undertaken on the authors' behalf by BCCM/LMG. DNA was prepared from strains CP6 and CP6e, Serratia plymuthica CIP $103239^{\mathrm{T}}$, Serratia grimesii CIP $103361^{\mathrm{T}}$, S. liquefaciens CIP $103238^{\mathrm{T}}$,
Serratia proteamaculans subsp. proteamaculans CIP $103236^{\mathrm{T}}$, S. proteamaculans subsp. quinovora LMG $7887^{\mathrm{T}}$ and Serratia fonticola $\mathrm{LMG} 8772^{\mathrm{T}}$ using the methods of Pitcher et al. (1989) and Wilson (1987).

Two DNA-DNA cross-hybridization methods were used. Strains CP6 and CP6e, S. plymuthica, S. grimesii, $S$. liquefaciens, $S$. proteamaculans subsp. proteamaculans and $S$. fonticola were cross-hybridized with each other using the fluorimetric method of Ezaki et al. (1989). Hybridizations were performed under high-stringency conditions in $50 \%$ formamide at $48{ }^{\circ} \mathrm{C}$. Strains CP6 and CP6e, $S$. proteamaculans subsp. proteamaculans and $S$. proteamaculans subsp. quinovora were cross-hybridized with each other using the initial renaturation method of De Ley et al. (1970), undertaken at $75^{\circ} \mathrm{C}$. All hybridizations were performed in both directions and in duplicate and the mean percentage DNA relatedness values were determined.

Statistical analysis of data. Statistics were performed with the MINITAB version 12 computer package. Mean phage titres were compared by analysis of variance after $\log _{10}$ transformation and the minimum significant difference (MSD) between means was calculated (Fry, 1993). Bacteriocin profiles of the various bacteria were investigated by cluster analysis. Jaccard's coefficients $\left(S_{\mathrm{j}}\right)$ were calculated by hand. Average linkage clustering (UPGMA) was then applied to the resulting distance matrix using the PHYLIP package.

The phenotypic profiles of bacteria from API strips and BiOLOG plates were also analysed by cluster analysis. The positive/negative scorings from both tests were pooled for each bacterium tested, a simple matching coefficient $\left(S_{\mathrm{sm}}\right)$ matrix was constructed from the resulting binary dataset and a cluster analysis was performed with UPGMA.

\section{RESULTS AND DISCUSSION}

\section{Initial screening for CP6-like bacteria}

Sugar beets were sampled 25, 52 and 82 days after sowing. Overall, $0.045 \%$ of the viable bacteria counted were CP6-like and 78.6\% of these putative CP6-like bacteria $(n=262)$ possessed the correct morphology when grown on TSBA and were therefore investigated further. Of these, $84.0 \%$ had varying degrees of sensitivity to the CP6-phage lysates. Seven patterns of phage sensitivity were identified, ranging from successful infection by most of the phages to insensitivity to all phages. A representative of each of these seven groups (CP6a-CP6g) was selected for subsequent analysis.

CP6-like bacteria were also isolated from samples taken from Oxleas wood in Kent and from scrubland in Cornwall. Although morphologically identical, these had phage-sensitivity patterns different from any of those identified from the Wytham Farm isolates. Isolates Ox2 (from Oxleas wood) and PC1 (from the earthworm) were chosen to represent these bacterial strains.

Finally, two further isolates, named RK and Ox1, were also selected for further investigation. Isolate RK was one of several isolates from stored 1996 sugar-beet samples from Wytham Farm which appeared CP6-like in all respects except for its red colony pigmentation on 
Table 2. Sensitivity of environmental isolates and selected type and reference strains to various CP6 bacteriophages

Values are means of two replicate titrations. The MSD (Fry, 1993) between $\log _{10}$ means at $P=0 \cdot 05$ was $0 \cdot 521$. L, Lysis characteristic of bacteriocins. Not all of the lysis observed was due to successful phage infection. Frequently, neat lysates would lyse bacterial lawns but, when diluted, their lytic effect would disappear completely rather than resolve into individual plaques, indicating bacteriocin, rather than phage, activity. - , No lysis observed. Isolate Ox1, S. fonticola IB4r and $H$. alvei NCTC $8105^{\mathrm{T}}$ were insensitive to all the phages tested. Furthermore, S. proteamaculans subsp. proteamaculans CIP $103236^{\mathrm{T}}$ and $S$. grimesii CIP $103361^{\mathrm{T}}$ had the same sensitivity as $S$. liquefaciens CIP $103238^{\mathrm{T}}$.

\begin{tabular}{|c|c|c|c|c|c|c|c|c|}
\hline \multirow[t]{2}{*}{ Strain } & \multicolumn{8}{|c|}{ Mean titre $\left[\log _{10}\right.$ (p.f.u. $\left.\left.\mathrm{ml}^{-1}\right)\right]$} \\
\hline & ФСР6-1 & ФСР6-2 & ФСР6-3 & ФСР6-4 & ФСР6-5 & ФСР6-6 & ФСР6-8 & ФСР6-9 \\
\hline \multicolumn{9}{|l|}{ Environmental isolates } \\
\hline CP6 & $9 \cdot 256$ & $9 \cdot 528$ & $9 \cdot 580$ & $9 \cdot 996$ & $9 \cdot 153$ & $7 \cdot 971$ & $\mathrm{~L}$ & - \\
\hline CP6a & $8 \cdot 860$ & $9 \cdot 054$ & $\mathrm{~L}$ & $9 \cdot 946$ & $8 \cdot 942$ & $8 \cdot 023$ & $\mathrm{~L}$ & - \\
\hline CP6b & $\mathrm{L}$ & $\mathrm{L}$ & $\mathrm{L}$ & $9 \cdot 317$ & $\mathrm{~L}$ & $\mathrm{~L}$ & $9 \cdot 662$ & - \\
\hline CP6c & $\mathrm{L}$ & $\mathrm{L}$ & $\mathrm{L}$ & $\mathrm{L}$ & $\mathrm{L}$ & $6 \cdot 384$ & - & - \\
\hline CP6d & $\mathrm{L}$ & $\mathrm{L}$ & $\mathrm{L}$ & $\mathrm{L}$ & $\mathrm{L}$ & $\mathrm{L}$ & - & $10 \cdot 042$ \\
\hline CP6e & - & - & - & - & - & - & $\mathrm{L}$ & $10 \cdot 190$ \\
\hline CP6f & - & - & $\mathrm{L}$ & $8 \cdot 314$ & - & $\mathrm{L}$ & - & - \\
\hline CP6g & - & - & $\mathrm{L}$ & - & - & - & $\mathrm{L}$ & - \\
\hline $\mathrm{PC} 1$ & $\mathrm{~L}$ & $\mathrm{~L}$ & $\mathrm{~L}$ & $\mathrm{~L}$ & $\mathrm{~L}$ & $\mathrm{~L}$ & - & - \\
\hline $\mathrm{Ox} 2$ & $\mathrm{~L}$ & $\mathrm{~L}$ & $\mathrm{~L}$ & $\mathrm{~L}$ & $\mathrm{~L}$ & $9 \cdot 150$ & $6 \cdot 688$ & - \\
\hline RK & $\mathrm{L}$ & $\mathrm{L}$ & $\mathrm{L}$ & $\mathrm{L}$ & $\mathrm{L}$ & $\mathrm{L}$ & - & $9 \cdot 872$ \\
\hline \multicolumn{9}{|l|}{ Type and reference strains } \\
\hline S. marcescens NCIB 1377 & - & - & - & - & - & $\mathrm{L}$ & - & - \\
\hline S. ficaria NCTC $12148^{\mathrm{T}}$ & - & - & - & - & - & $\mathrm{L}$ & $\mathrm{L}$ & $8 \cdot 302$ \\
\hline S. liquefaciens CIP $103238^{\mathrm{T}}$ & $\mathrm{L}$ & $\mathrm{L}$ & $\mathrm{L}$ & $\mathrm{L}$ & $\mathrm{L}$ & $\mathrm{L}$ & - & - \\
\hline $\begin{array}{l}\text { S. proteamaculans subsp. } \\
\text { quinovora } \mathrm{LMG} 7887^{\mathrm{T}}\end{array}$ & $\mathrm{L}$ & $\mathrm{L}$ & $\mathrm{L}$ & $4 \cdot 477$ & $\mathrm{~L}$ & $\mathrm{~L}$ & $\mathrm{~L}$ & - \\
\hline S. plymuthica CIP $103239^{\mathrm{T}}$ & $\mathrm{L}$ & $\mathrm{L}$ & $\mathrm{L}$ & $\mathrm{L}$ & $\mathrm{L}$ & $9 \cdot 444$ & - & $8 \cdot 710$ \\
\hline
\end{tabular}

TSBA when incubated at less than $30^{\circ} \mathrm{C}$. Isolate RK was notable in showing definite sensitivity to the lysate of phage ФСР6-9. In contrast, Ox1, an isolate from Oxleas wood, was typical of isolates that appeared CP6-like on SSM but did not show CP6-like colony morphology when grown on TSBA and were insensitive to all of the phages.

\section{Quantifying phage sensitivity of environmental isolates and type and reference strains}

All 11 isolates described above, the original CP6 strain and various type and reference strains were titrated against all the phages in our collection. The resulting mean titres (Table 2) showed that, not only were the various CP6-like isolates sensitive to different combinations of phages, but there was significant variation in sensitivity. For example, isolates CP6, CP6a, CP6b and CP6f were all sensitive to phage ФСР6-4, yet CP6f was significantly less sensitive than $\mathrm{CP} 6 \mathrm{~b}$, which in turn was significantly less sensitive than CP6 and CP6a (Table 2). This variation in sensitivity frequently coincided with variations in plaque morphology. Thus, ФСР6-4 produced large, clear plaques surrounded by several concentric rings on CP6 (Ashelford et al., 1999b), smaller plaques without rings on CP6a and tiny, barely discernible plaques on CP6b and CP6f.
Type strains of $S$. proteamaculans subsp. quinovora, Serratia ficaria and S. plymuthica were also susceptible to some of the phages tested, as was the red-pigmented isolate, RK (Table 2). All other strains were insensitive to successful phage infection, although considerable amounts of non-specific cell lysis were observed.

\section{Bacteriocin production and sensitivity of isolates and type and reference strains}

Most of the environmental isolates produced detectable bacteriocins, as did many of the type and reference strains tested (a full table of data is available as supplementary material in IJSEM Online at http://ijs.sgmjournals.org/). Bacteriocin production was most noticeable when supernatants were harvested from bacteria grown at $20^{\circ} \mathrm{C}$ rather than $30{ }^{\circ} \mathrm{C}$. There was a large variation in the degree of lysis observed, ranging from total clearing of the bacterial lawn to turbid zones that were only just discernible. On only two occasions was this lysis the result of phage infection. This was when CP6g was inoculated with neat supernatant from CP6d and when CP6d was inoculated with neat supernatant from the $S$. proteamaculans subsp. proteamaculans type strain. On both of these occasions, a faint scattering of tiny plaques was seen. These plaques were few in number 


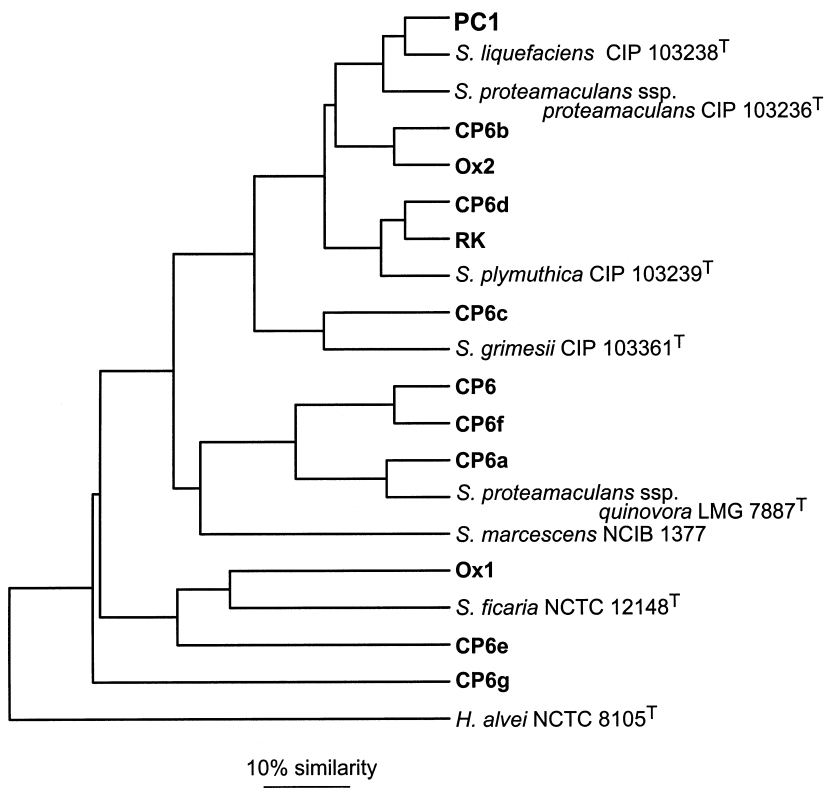

Fig. 1. Cluster analysis of bacteria according to bacteriocin sensitivity. A distance matrix of Jaccard's coefficients $\left(S_{j}\right)$ was clustered according to the UPGMA algorithm. Other treeconstruction methods gave similar tree topologies.

and were barely discernible. In all other cases, serial dilution of the lytic supernatants failed to resolve the observed zones of lysis into individual plaques; rather, the lysis effect disappeared between successive dilutions, a phenomenon characteristic of bacteriocin activity (Day, 1997).

All the bacteria, with the exception of Hafnia alvei, were sensitive to at least some of the bacteriocins produced, and each bacterium tested had a different sensitivity profile. A cluster analysis of these data is presented in Fig. 1.

\section{Probing whole-genome extracts with DIG-labelled phage DNA}

To assay prophages, total genomic bacterial DNA was probed with DIG-labelled DNA from phages ФCP6-1 to -6. Positive cross-hybridizations were only observed for the four lysogen controls, the CP6-like isolate Ox2, $S$. fonticola IB4r and the $S$. liquefaciens and $S$. proteamaculans subsp. quinovora type strains. A very weak hybridization signal was also obtained with the type strain of $S$. grimesii.

\section{$16 \mathrm{~S}$ rRNA gene sequence analysis}

The cloned 16S rRNA gene from strain CP6 was sequenced three times in both directions and the resulting sequences were compared to assess sequencing error. Sequences varied by 9 bases at most prior to editing and this resulted in an uncorrected evolutionary distance of $0.2 \%$ in the edited dataset. A consensus sequence of $1360 \mathrm{bp}$ was constructed (ac- cession no. AJ279044) and compared with sequences generated (sequenced once in both directions) from the other isolates along with those from the DDBJ/ EMBL/GenBank databases.

Sequences from isolates CP6a-CP6d, CP6g and PC1 showed similarities of $99 \cdot 7-99.9 \%$ towards CP6 and were thus unambiguously confirmed to be members of the same species. The CP6f sequence was $99.5 \%$ similar, showing that it too was most likely to belong to the same species as CP6. Isolate CP6e showed the greatest divergence, at $99 \cdot 2 \%$ similarity $(14$ bases different before editing). The non-CP6-like isolates RK and Ox1 were respectively 1.2 and $2.1 \%$ distant, corresponding to 21 and 33 unedited base differences from CP6.

Comparisons with sequences from the public databases revealed the $\mathrm{CP} 6$ sequence to be almost identical to that of the $S$. proteamaculans subsp. quinovora type strain $(99 \cdot 9 \%$ similarity). The next closest sequence was from $S$. proteamaculans subsp. proteamaculans DSM $4543^{\mathrm{T}}(99 \cdot 5 \%)$ followed by $S$. grimesii DSM $30063^{\mathrm{T}}(99 \cdot 3 \%)$. This order of similarity was repeated for all the CP6-like bacterial sequences with the exception of CP6e, which was most similar to $S$. proteamaculans subsp. proteamaculans DSM $4543^{\mathrm{T}}$ $(99 \cdot 7 \%)$, then $S$. grimesii DSM $30063^{\mathrm{T}}(99 \cdot 5 \%)$ and only then $S$. proteamaculans subsp. quinovora DSM $4597^{\mathrm{T}}(99 \cdot 2 \%)$. Of the non-CP6-like bacteria, the redpigmented isolate RK was closest to $S$. plymuthica DSM $4540^{\mathrm{T}}(99.8 \%)$ and, equally, isolate Ox1 was closer to $S$. fonticola DSM $4576^{\mathrm{T}}(99 \cdot 5 \%)$ than any other sequence.

All but one of the CP6-like bacteria clustered unambiguously with $S$. proteamaculans subsp. quinovora DSM $4597^{\mathrm{T}}$. The exception, CP6e, clustered with $S$. proteamaculans subsp. proteamaculans DSM $4543^{\mathrm{T}}$ and $S$. grimesii DSM $30063^{\mathrm{T}}$ (Fig. 2). Isolate RK grouped closely with $S$. plymuthica DSM $4540^{\mathrm{T}}$, whilst Ox1 clustered unequivocally with $S$. fonticola DSM $4576^{\mathrm{T}}$. Other tree-constructing methods were employed to test the rigour of these groupings (data not shown). Although the overall topology of the genus Serratia sometimes varied in these trees, all but the unrooted parsimony method of Felsenstein (1993) were consistent in clustering the environmental isolates with the same type strains as seen in Fig. 2.

Sequence analysis of $16 \mathrm{~S}$ rRNA genes was very consistent in identifying CP6 and all but one (CP6e) isolate as $S$. proteamaculans subsp. quinovora, despite its frequent unreliability for distinguishing bacteria beyond the genus level (Fox et al., 1992; Wayne et al., 1987). However, this was only achieved once reliable sequences for all the known Serratia species had become available from the DDBJ/EMBL/GenBank databases (after Spröer et al., 1999), highlighting that this form of identification can only be as good as the public database upon which it relies.

Considering the genus Serratia as a whole, our $16 \mathrm{~S}$ rRNA gene sequence phylogenetic tree (Fig. 2) shows 


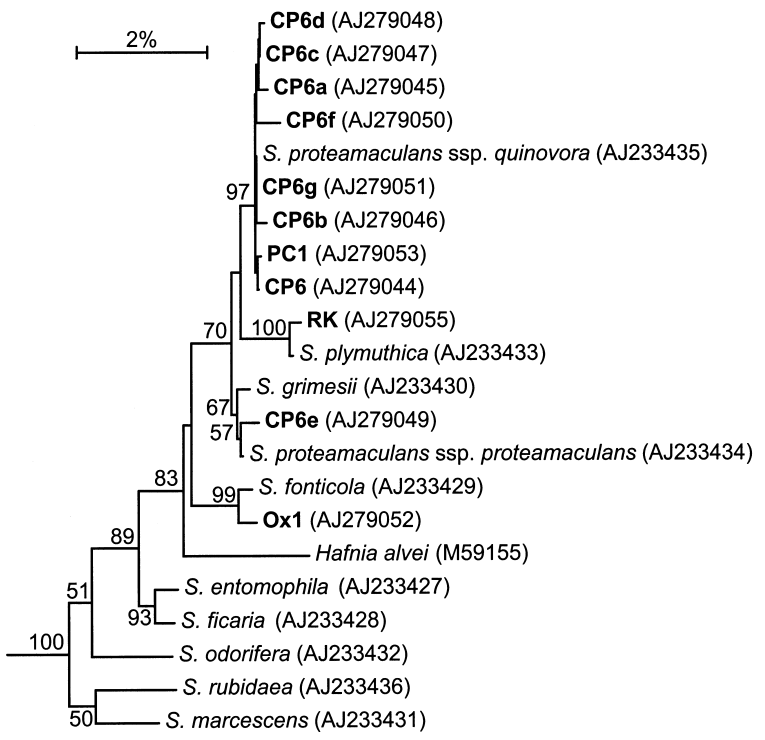

Fig. 2. Phylogenetic reconstruction of the genus Serratia based on edited 16S rRNA sequence data (1312 bp) illustrating the phylogeny of the environmental strains isolated during this study, highlighted in bold. Sequences for type strains were from Spröer et al. (1999). The tree was constructed from a Jukes and Cantor distance matrix using the neighbour-joining method and was rooted with Buchnera aphidicola (accession no. L18927; not shown). The dataset was bootstrapped 100 times; only bootstrap values $50 \%$ or greater are shown. Accession numbers for sequences are shown in parentheses. Bar, 2 substitutions per 100 nucleotides.

$H$. alvei splitting the genus Serratia; other enterobacterial genera (Obesumbacterium, Rahnella, Yersinia, Ewingella, Budvicia, Pragia and Leminorella) also split the genus Serratia in this manner. This genussplitting phenomenon is consistent with the 16S RNA gene sequence trees published by Spröer et al. (1999) and Dauga (2002). However, using gyr B sequences, Dauga (2002) found that all the Serratia species formed a monophyletic group supported by high bootstrap values and phenotypic information. So, 16S rRNA gene sequences do not give an accurate picture of the more deeply branching phylogenies within Enterobacteriaceae.

\section{Whole-genome DNA-DNA cross-hybridization}

Isolate CP6 shared $100 \%$ DNA relatedness with $S$. proteamaculans subsp. quinovora, conclusively identifying CP6. Consistent with this identification, isolate CP6 exhibited DNA relatedness values with the other type strains at values well below the value of $70 \%$ required to signify species identity (Wayne et al., 1987). A table with full results for all the DNA-DNA cross-hybridizations carried out is available as supplementary material in IJSEM Online (http:// ijs.sgmjournals.org/).

Isolate CP6e was the only CP6-like bacterium that failed to cluster closely with CP6 during 16S rRNA

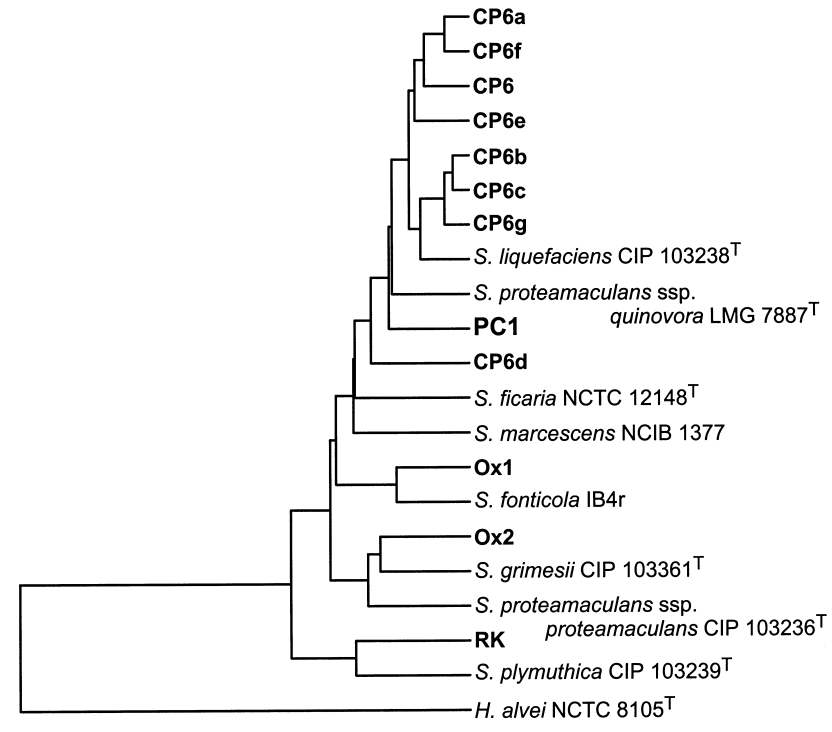

$10 \%$ similarity

Fig. 3. Cluster analysis of bacteria according to API $20 \mathrm{E}$ and BiOLOG profiles. A distance matrix of simple similarity coefficients $\left(S_{\mathrm{sm}}\right)$ was clustered with the UPGMA algorithm. Other tree-construction methods gave similar tree topologies.

sequence analysis. The mean DNA-DNA relatedness between isolates CP6 and CP6e was $88 \%$, so they are members of the same species, in spite of the 16S rDNA sequence analysis. Moreover, CP6e showed greater than $70 \%$ relatedness with $S$. proteamaculans subsp. quinovora $(85 \%)$ and less than $70 \%$ relatedness with $S$. proteamaculans subsp. proteamaculans (63-68\%). Cross-hybridizations amongst the type strains were consistently less than $70 \%$ similar $(19-57 \%)$; this included the two $S$. proteamaculans subspecies (46-68\%).

\section{Identification methods based on phenotype}

API 20E strips failed to identify many of the isolates, and those for which an identification was obtained could only be identified unambiguously at the genus level. Isolates $\mathrm{CP} 6 \mathrm{~d}, \mathrm{CP} 6 \mathrm{e}, \mathrm{PC} 1$ and $\mathrm{Ox} 2$ were tentatively identified as $S$. liquefaciens, with the percentage identities ranging from 73 to $76 \cdot 2 \%$. Isolate RK was identified as either Serratia rubidaea or $S$. plymuthica at 46 and $42.6 \%$, respectively. Isolate Ox 1 was identified as $S$. fonticola at $31 \cdot 6 \%$. Using BiOLOG plates, all but one of the CP6-like bacteria were identified as $S$. liquefaciens $/ S$. grimesii, with similarity values ranging from 0.548 to 0.970 . The exception was CP6b, being identified as Serratia marcescens $(0 \cdot 743)$. Isolate RK was identified as $S$. plymuthica at $0 \cdot 610$, whilst Ox1 was identified as $S$. fonticola at 0.468. A cluster analysis of the combined API $20 \mathrm{E}$ and BiOLOG profiles was attempted to determine whether identities could be further clarified (Fig. 3). All but one of the CP6-like isolates clustered closely with $S$. liquefaciens 
CIP $103238^{\mathrm{T}}$ and S. proteamaculans subsp. quinovora LMG $7887^{\mathrm{T}}$, the exception being isolate Ox2. A cluster analysis of the FAME profiles of CP6a-CP6g with CP6 indicated they were the same (data not shown). Like CP6 (similarity coefficient, 0.774), they were identified by FAME analysis as $S$. liquefaciens $/ S$. proteamaculans (Ashelford et al., 1999a).

Overall, these three phenotypic identification methods were unreliable for natural isolates of Serratia spp. API performed least well and BiOLOG proved more effective, with positive identifications obtained regularly. Furthermore, the dendrogram of the BiOLOG and API data was not consistent with the 16S rDNA trees and DNA-DNA hybridization results.

Isolates CP6 and CP6e were identified using Biotype 100 strips. CP6 was identified as $S$. proteamaculans subsp. quinovora, whilst CP6e was identified as $S$. proteamaculans subsp. proteamaculans biotype C1c. Hence, based on the two results obtained, this proved the most accurate phenotypic method.

\section{Ecological implications}

Our CP6 isolates were distinguishable from one another in two ecologically significant ways. They varied both in their sensitivity to indigenous phages and in their sensitivity to, and production of, bacteriocins. These differences are not remarkable, as medical strains can be classified by their bacteriocin profiles (Farmer, 1972a, b) and 'phage-typed' with suites of characterized phages. However, our bacteriocin and phage-sensitivity results do not correlate with the phylogeny of the Serratia species and so cannot be used for identifying these bacteria. Almost all these isolates came from the same environment as the original phage collection. Our previous investigations have demonstrated that these phages show distinct, different temporal population fluctuations and competition within the rhizosphere (Ashelford et al., 1999a, 2000). Now we have shown that the bacterial species on which they predate is diverse. Collectively, our results imply a complex underlying interaction amongst bacterial species and with predatory phages in situ.

The bacteriocin diversity profiles observed demonstrate that these CP6 strains and related species have the potential to interact antagonistically amongst themselves in situ. It is probable that the bacteriocin activity derives from previous phage infections, either through the creation of a viable lysogenic relationship with the host or by leaving a disabled prophage within the genome. Some Serratia bacteriocins are prophage derived (Prinsloo, 1966), and we have observed phage tail-like structures within CP6 supernatant (data not shown) that cannot be accounted for by known phage infections and could explain the lytic effects observed. Similar phage-tail-like pyocins in Pseudomonas aeruginosa have been shown recently (Nakayama et al., 2000) to be phage tails that have been evolutionarily specialized as bacteriocins and not simply defective phages. The absence of prophages from all our Wytham Farm isolates indicates that the same might be true for these bacteria.

Our results also show that the host ranges of the CP6 phages were not restricted to a single species. Titration experiments showed that some of these phages could also infect $S$. plymuthica (both the indigenous isolate RK and the type strain) and the S. ficaria type strain successfully (Table 2). We have no evidence that these phages are capable of infecting bacteria beyond the genus Serratia. However, other work has shown that Serratia-infecting phages can infect non-Serratia members of the Enterobacteriaceae (Prinsloo, 1966). There are also some descriptions in the literature of broad-host-range phages that infect other genera and that have been isolated from barley rhizosphere (Campbell et al., 1995) and aquatic environments (Matsuzaki et al., 1992; Jensen et al., 1998).

One way in which the complex interaction between phage and bacteria may manifest itself is through horizontal gene transfer, leading to a mixing of the genetic pool that makes phylogenetic determination more difficult. The cluster analysis of our bacteriocin data (Fig. 1) showed that bacteriocin interactions do not follow phylogeny. If the observed bacteriocin activity was the consequence of earlier phage infections, especially as the phages have broad host ranges, this should not be surprising. Horizontal gene transfer may go some way to explain the difficulties in resolving the phylogeny of genera such as Serratia.

\section{Taxonomy of the S. proteamaculans subspecies}

A combination of $16 \mathrm{~S}$ rRNA sequence analysis and DNA-DNA cross-hybridization clearly demonstrated that all our CP6-like isolates and PC1 were members of the same species as $S$. proteamaculans subsp. quinovora. Strain Ox2 is most likely to be a member of $S$. proteamaculans subsp. quinovora, based on its bacteriophage sensitivity (Table 2), although not all the key BiOLOG test results were consistent with those from the other strains of this subspecies (Table 3). However, isolates RK and Ox1 were respectively readily identified as $S$. plymuthica and $S$. fonticola for the following reasons, and so were not true CP6-like isolates. The high $(>99.5 \%)$ 16S rDNA sequence similarity between these isolates and the closest type strains indicate that the DNA-DNA genomic hybridization frequencies between these bacteria are highly likely to be $>70 \%$ (Keswani \& Whitman, 2001). Isolate RK had red colonies on TSBA and both isolates had BiOLOG substrate utilization profiles similar to those expected for their identities (Fig. 3).

Our DNA-DNA hybridization results are consistent with those of Grimont et al. (1982a). Taking both these sets of results together, it is clear that all strains of $S$. proteamaculans subsp. quinovora show $>80 \%$ relatedness $(n=16)$ and so form a more coherent species by DNA-DNA hybridization than any of the other $S$. liquefaciens-like species investigated by 
Table 3. Characteristics useful in differentiating $S$. quinivorans sp. nov. isolates from $S$. proteamaculans

Strains are designated as: $1, S$. quinivorans strains CP6, CP6a, CP6b, CP6c, CP6e, CP6f, CP6g, PC1 and LMG 7887' ; 2, S. quinivorans $\mathrm{CP} 6 \mathrm{~d} ; 3$, S. quinivorans $\mathrm{Ox} 2 ; 4$, S. proteamaculans subsp. proteamaculans CIP $103236^{\mathrm{T}}$.

\begin{tabular}{|lcccc|}
\hline Characteristic & $\mathbf{1}$ & $\mathbf{2}$ & $\mathbf{3}$ & $\mathbf{4}$ \\
\hline Metabolism of:* & & & & \\
$\quad$ Inositol & & & & \\
Sucrose & + & + & + & - \\
Growth on: $\dagger$ & + & + & + & - \\
Cellobiose & & & & \\
L-Rhamnose & + & - & + & - \\
D-Galactonic acid lactone & + & + & - & - \\
D-Gluosaminic acid & + & + & + & - \\
D-Glucuronic acid & + & + & - & - \\
Hydroxy-L-proline & + & + & + & - \\
\hline
\end{tabular}

* From API 20E strips incubated at $30^{\circ} \mathrm{C}$ for $24 \mathrm{~h}$.

$\dagger$ From BiOLOG GN microplates incubated at $30^{\circ} \mathrm{C}$ for $24 \mathrm{~h}$.

Grimont et al. (1982a). S. proteamaculans subsp. quinovora is also distinct from subsp. proteamaculans, as these two studies show that $90 \%(n=42)$ of the DNA-DNA hybridizations between these two subspecies showed $<70 \%$ DNA relatedness. $S$. proteamaculans subsp. proteamaculans is also not as coherent as subsp. quinovora, because $16 \%(n=38)$ of the hybridizations of strains within subsp. proteamaculans showed $<70 \%$ but $>60 \%$ DNA-DNA relatedness. In addition, Grimont et al. (1982b) were able to distinguish the two $S$. proteamaculans subspecies phenotypically with biochemical tests. So, we propose that $S$. proteamaculans subsp. quinovora be transferred to a novel species, Serratia quinivorans corrig., sp. nov. The type strain for $S$. quinivorans $\mathrm{sp}$. nov. should remain the same as previously designated for $S$. proteamaculans subsp. quinovora $\left(\mathrm{CIP} 8195^{\mathrm{T}}=\right.$ $\operatorname{ATCC} 33765^{\mathrm{T}}=\mathrm{LMG} 7887^{\mathrm{T}}$ ).

\section{Distinguishing S. quinivorans sp. nov. from $S$. proteamaculans and the other Serratia species}

A combination of $16 \mathrm{~S}$ rRNA gene sequence analysis and DNA-DNA hybridization is the best way to distinguish members of this species group. BiOLOG GN microplate and Biotype 100 identification can provide a useful first step, although, as discussed previously, neither system gives definitive results for all Serratia species. Table 3 gives the BiOLOG tests, after incubation at $30{ }^{\circ} \mathrm{C}$ for $24 \mathrm{~h}$, that provided definitive separation between all the strains of $S$. quinivorans sp. nov. that we tested and the $S$. proteamaculans type strain. The lack of both aesculin hydrolysis (API CH) after 2 days and acid production from salicin in 5 days also distinguishes $S$. quinivorans sp. nov. from all the other S. liquefaciens-like species (Grimont et al., 1982b). Further tests for distinguishing Serratia species are provided elsewhere (Grimont et al., 1982b; Grimont \& Grimont, 1984, 1992).

Grimont et al. (1982b) gave growth on quinic acid as the sole carbon source at $30^{\circ} \mathrm{C}$ in 14 days as a distinguishing test between $S$. quinivorans sp. nov. and other $S$. liquefaciens-like species, except for $S$. plymuthica. However, although all our strains of $S$. quinivorans sp. nov. grew on M70 quinic acid plates in 14 days (colony size $0.4-0.8 \mathrm{~mm}$ ), so did the type strains of $S$. liquefaciens and $S$. proteamaculans, with the same colony size. The type strain of $S$. plymuthica and isolate RK grew more strongly (colony size after 14 days $1.2-1.7 \mathrm{~mm}$ ), but the type strain of $S$. grimesii did not grow at all. This test is therefore only useful for distinguishing $S$. quinivorans sp. nov. from $S$. grimesii. In all cases, growth was apparent after $48 \mathrm{~h}$ and relative colony sizes distinguishable by 7 days.

\section{Description of Serratia quinivorans corrig., sp. nov.}

Serratia quinivorans (qui.ni.vor'ans. N.L. n. acidum quinicum quinic acid; L. v. vorare to devour; N.L. fem. part. adj. quinivorans devouring quinic acid).

Originally classified as $S$. proteamaculans subsp. quinovora. Cells are straight, Gram-negative, nonspore-forming rods, $0.5-0.8 \mu \mathrm{m}$ wide by $0.9-2.0 \mu \mathrm{m}$ long with rounded ends, that are motile by means of peritrichous flagella. Facultatively anaerobic, growing aerobically on nutrient agar in $24 \mathrm{~h}$ at $30^{\circ} \mathrm{C}$ as colonies that are $1-3 \mathrm{~mm}$ in diameter, circular, shiny, domed, opaque, cream-white and smooth with an entire margin, having a distinctive musty odour like other Serratia species. Does not require growth factors. Grows on a wide range of carbon sources including glucose, sucrose, quinic acid and, for most strains, trans-aconitate, cellobiose, malate and L-rhamnose, but not adonitol or benzoate. Most strains produce lysine decarboxylase, ornithine decarboxylase, chitinase and gelatinase, but not arginine decarboxylase. Aesculin is not hydrolysed within 2 days but is within 7 days. Acid is produced from glucose and triacetin but not from lactose or salicin. Full details of other phenotypic tests are available elsewhere (Grimont et al., 1982b; Grimont \& Grimont, 1984, 1992). Occurs in soil, in association with plants and in the digestive tracts of soil invertebrates. The $\mathrm{G}+\mathrm{C}$ content of the DNA is $53-54 \mathrm{~mol} \%$ and the type strain is strain $4123^{\mathrm{T}}$ $\left(=\mathrm{CIP} \quad 8195^{\mathrm{T}}=\mathrm{ATCC} \quad 33765^{\mathrm{T}}=\mathrm{LMG} \quad 7887^{\mathrm{T}}\right)$ (Grimont et al., 1982a, b).

\section{ACKNOWLEDGEMENTS}

This work was supported by a Ministry of Agriculture Fisheries and Food (UK) grant, RG0112.

\section{REFERENCES}

Adams, M. H. (1959). Bacteriophages. New York: Interscience Publishers. 
Ashelford, K. E., Day, M. J., Bailey, M. J., Lilley, A. K. \& Fry, J. C. (1999a). In situ population dynamics of bacterial viruses in a terrestrial environment. Appl Environ Microbiol 65, 169-174.

Ashelford, K. E., Fry, J. C., Bailey, M. J., Jeffries, A. R. \& Day, M. J. (1999b). Characterization of six bacteriophages of Serratia liquefaciens CP6 isolated from the sugar beet phytosphere. Appl Environ Microbiol 65, 1959-1965.

Ashelford, K. E., Norris, S. J., Fry, J. C., Bailey, M. J. \& Day, M. J. (2000). Seasonal population dynamics and interactions of competing bacteriophages and their host in the rhizosphere. Appl Environ Microbiol 66, 4193-4199.

Ashelford, K. E., Learner, M. A. \& Fry, J. C. (2001). Gene transfer and plasmid instability within pilot-scale sewage filter beds and the invertebrates that live in them. FEMS Microbiol Ecol 35, 197-205.

Campbell, J. I. A., Albrechtsen, M. \& Sørensen, J. (1995). Large Pseudomonas phages isolated from barley rhizosphere. FEMS Microbiol Ecol 18, 63-74.

Dauga, C. (2002). Evolution of the gyrB gene and the molecular phylogeny of Enterobacteriaceae: a model molecule for molecular systematic studies. Int J Syst Evol Microbiol 52, 531-547.

Day, M. J. (1997). Bacteriophage and bacteriocins. In Topley and Wilson's Microbiology and Microbial Infections, pp. 185-194. Edited by A. Balows \& B. I. Duerden. London: Edward Arnold.

Day, M. J. \& Marchesi, J. R. (1996). Transduction in the aquatic environment. In Molecular Microbial Ecology Manual, pp. 5.3.1.1-5.3.1.21. Edited by A. D. L. Akkermans, J. D. van Elsas \& F. J. de Bruijn. Dordrecht: Kluwer.

De Ley, J., Cattoir, H. \& Reynaerts, A. (1970). The quantitative measurement of DNA hybridization from renaturation rates. Eur $J$ Biochem 12, 133-142.

Ezaki, T., Hashimoto, Y. \& Yabuuchi, E. (1989). Fluorometric deoxyribonucleic acid-deoxyribonucleic acid hybridization in microdilution wells as an alternative to membrane filter hybridization in which radioisotopes are used to determine genetic relatedness among bacterial strains. Int J Syst Bacteriol 39, 224-229.

Farmer, III, J. J. (1972a). Epidemiological differentiation of Serratia marcescens: typing by bacteriocin production. Appl Microbiol 23, 218-225.

Farmer, III, J. J. (1972b). Epidemiological differentiation of Serratia marcescens: typing by bacteriocin sensitivity. Appl Microbiol 23, 226-231.

Felsenstein, J. (1993). PHYLIP (Phylogeny Inference Package) version 3.5c. Distributed by the author. Department of Genetics, University of Washington, Seattle, USA.

Fox, G. E., Wisotzkey, J. D. \& Jurtshuk, P., Jr (1992). How close is close: $16 \mathrm{~S}$ rRNA sequence identity may not be sufficient to guarantee species identity. Int J Syst Bacteriol 42, 166-170.

Fry, J. C. (1993). Biological Data Analysis: a Practical Approach. Oxford: IRL Press.

Grimont, P. A. D. \& Grimont, F. (1984). Genus VIII. Serratia Bizio $1823,288^{\mathrm{AL}}$. In Bergey's Manual of Systematic Bacteriology, vol. 1, pp. 477-484. Edited by N. R. Krieg \& J. G. Holt. Baltimore: Williams \& Wilkins.

Grimont, F. \& Grimont, P. A. D. (1992). The genus Serratia. In The Prokaryotes, pp. 2822-2848. Edited by A. Balows, H. G. Trüper, M. Dworkin, W. Harder \& K.-H. Schleifer. New York: Springer.

Grimont, P. A. D., Grimont, F. \& Starr, M. P. (1981). Serratia species isolated from plants. Curr Microbiol 5, 317-322.

Grimont, P. A. D., Irino, K. \& Grimont, F. (1982a). The Serratia liquefaciens-S. proteamaculans-S. grimesii complex: DNA relatedness. Curr Microbiol 7, 63-68.
Grimont, P. A. D., Grimont, F. \& Irino, K. (1982b). Biochemical characterization of Serratia liquefaciens sensu stricto, Serratia proteamaculans, and Serratia grimesii sp. nov. Curr Microbiol 7, 69-74.

Jensen, E. C., Schrader, H. S., Rieland, B., Thompson, T. L., Lee, K. W., Nickerson, K. W. \& Kokjohn, T. A. (1998). Prevalence of broad-host-range lytic bacteriophages of Sphaerotilus natans, Escherichia coli, and Pseudomonas aeruginosa. Appl Environ Microbiol 64, 575-580.

Kalbe, C., Marten, P. \& Berg, G. (1996). Strains of the genus Serratia as beneficial rhizobacteria of oilseed rape with antifungal properties. Microbiol Res 151, 433-439.

Keswani, J. \& Whitman, W. B. (2001). Relationship of 16S rRNA sequence similarity to DNA hybridization in prokaryotes. Int $J$ Syst Evol Microbiol 51, 667-678.

Marchesi, J. R., Sato, T., Weightman, A. J., Martin, T. A., Fry, J. C., Hiom, S. J., Dymock, D. \& Wade, W. G. (1998). Design and evaluation of useful bacterium-specific PCR primers that amplify genes coding for bacterial 16S rRNA. Appl Environ Microbiol 64, 795-799.

Matsuzaki, S., Tanaka, S., Koga, T. \& Kawata, T. (1992). A broadhost-range vibriophage, KVP40, isolated from sea water. Microbiol Immunol 36, 93-97.

Nakayama, K., Takashima, K., Ishihara, H. \& 7 other authors (2000). The R-type pyocin of Pseudomonas aeruginosa is related to P2 phage, and the F-type is related to lambda phage. Mol Microbiol 38, 213-231.

Pitcher, D. G., Saunders, N. A. \& Owen, R. J. (1989). Rapid extraction of bacterial genomic DNA with guanidium thiocyanate. Lett Appl Microbiol 8, 151-156.

Powell, B. J., Purdy, K. J., Thompson, I. P. \& Bailey, M. J. (1993). Demonstration of tra + plasmid activity in bacteria indigenous to the phyllosphere of sugar beet; gene transfer to a recombinant pseudomonad. FEMS Microbiol Ecol 12, 195-206.

Prinsloo, H. E. (1966). Bacteriocins and phages produced by Serratia marcescens. J Gen Microbiol 45, 205-212.

Sambrook, J., Fritsch, E. F. \& Maniatis, T. (1989). Molecular Cloning : a Laboratory Manual, 2nd edn. Cold Spring Harbor, NY: Cold Spring Harbor Laboratory.

Spröer, C., Mendrock, U., Swiderski, J., Lang, E. \& Stackebrandt, E. (1999). The phylogenetic position of Serratia, Buttiauxella and some other genera of the family Enterobacteriaceae. Int J Syst Bacteriol 49, 1433-1438.

Thompson, J. D., Higgins, D. G. \& Gibson, T. J. (1994). Clustal W: improving the sensitivity of progressive multiple sequence alignment through sequence weighting, position-specific gap penalties and weight matrix choice. Nucleic Acids Res 22, 4673-4680.

Van de Peer, Y. \& De Wachter, R. (1994). TREECON for Windows: a software package for the construction and drawing of evolutionary trees for the Microsoft Windows environment. Comput Appl Biosci 10, 569-570.

Wayne, L. G., Brenner, D. J., Colwell, R. R. \& 9 other authors (1987). Report of the ad hoc committee on reconciliation of approaches to bacterial systematics. Int J Syst Bacteriol 37, 463-464.

Wilson, K. (1987). Preparation of genomic DNA from bacteria. In Current Protocols in Molecular Biology, pp. 2.4.1-2.4.5. Edited by F. M. Ausubel, R. Brent, R. E. Kingston, D. D. Moore, J. G. Seidman, J. A. Smith \& K. Struhl. New York: Wiley-Interscience.

Zhang, F., Dashti, N., Hynes, R. K. \& Smith, D. L. (1996). Plant growth promoting rhizobacteria and soybean [Glycine max (L.) Merr.] nodulation and nitrogen fixation at suboptimal root zone temperatures. Ann Bot 77, 453-459.

Zhang, F., Dashti, N., Hynes, R. K. \& Smith, D. L. (1997). Plant growth-promoting rhizobacteria and soybean [Glycine max (L.) Merr.] growth and physiology at suboptimal root zone temperatures. Ann Bot 79, 243-249. 